\title{
Neural correlates of the precedence effect in auditory evoked potentials
}

\author{
Jörg Damaschke *, Helmut Riedel, Birger Kollmeier \\ Medizinische Physik, Carl von Ossietzky Universität Oldenburg, D-26111 Oldenburg, Germany
}

Received 15 September 2004; accepted 14 March 2005

Available online 13 April 2005

\begin{abstract}
The precedence effect in subjective localization tasks reflects the dominance of directional information of a direct sound (lead) over the information provided by one or several reflections (lags) for short delays. By collecting data of both psychoacoustical measurements and auditory evoked potentials the current study aims at neurophysiological correlates for the precedence effect in humans. In order to investigate whether the stimulus features or the perception of the stimulus is reflected on the ascending stages of the human auditory pathway, auditory brainstem responses (ABRs) as well as cortical auditory evoked potentials (CAEPs) using double click-pairs were recorded. Potentials were related to the results of the psychoacoustical data.

ABRs to double click-pairs with lead-lag delays from 0 to $20 \mathrm{~ms}$ and interaural time differences (ITDs) in the lag click of 0 and $300 \mu$ s show an emerging second wave $V$ for lead-lag delays larger than $2 \mathrm{~ms}$. The amplitudes of the first and second wave $V$ are the same for a lead-lag delay of about $5 \mathrm{~ms}$. For the lag-ITD stimuli the latency of the second wave $V$ was prolonged by approximately ITD/2 compared to the stimuli without lag-ITD. As the amplitudes of the second wave $V$ were not decreased for a lead-lag delay around $5 \mathrm{~ms}$ as could be expected from psychoacoustical measurements of the precedence effect, ABRs reflect stimulus features rather than the perceptive qualities of the stimulus.

The mismatch negativity (MMN) component of the CAEP for double click-pairs was determined using a diotic standard and a deviant with an ITD of $800 \mu \mathrm{s}$ in the lag click. The comparison between the MMN components and the psychoacoustical data shows that the MMN is related to the perception of the stimulus, i.e., to the precedence effect.

Generally, the findings of the present study suggest that the precedence effect is not a result of a poor sensitivity of the peripheral bottom-up processing. Rather, the precedence effect seems to be reflected by the MMN, i.e., cognitive processes on higher stages of the auditory pathway.
\end{abstract}

(c) 2005 Elsevier B.V. All rights reserved.

Keywords: Precedence effect; Auditory evoked potential; Discrimination; Mismatch negativity

Abbreviations: ABR, auditory brainstem response; AC, auditory cortex; AVCN, anteroventral cochlear nucleus; CAEP, cortical auditory evoked potential; DCN, dorsal cochlear nucleus; EEG, electroencephalography; IC, inferior colliculus; ITD, interaural time difference; MMN, mismatch negativity; nHL, normal hearing level; SNR, signal-to-noise ratio; SOC, superior olivary complex

* Corresponding author. Fax: +49 4417983902.

E-mail address: joerg.damaschke@uni-oldenburg.de (J. Damaschke).

\section{Introduction}

If a sound is emitted in a reverberant environment, a complex mixture of acoustic signals comprising the direct sound (lead) and several reflections (lags) reaches the two ears. Although lead and lag sounds may carry contradictory directional information, the human auditory system manages well to resolve the location of the sound source. It is commonly believed that this ability 
is assisted by the precedence effect, a term that pools several phenomena which describe the dominance of directional information of a leading sound over directional information provided by lagging sounds for short delays.

The precedence effect was first described by Wallach et al. (1949). Since then, many researchers have shed light on the relationship between the information which is provided by the lead and the lag, respectively. For comprehensive reviews see, e.g., Zurek (1980), Blauert (1997) and Litovsky et al. (1999).

Single cell studies were made in different species, e.g., in the external colliculus of the inferior colliculus of the barn owl (Keller and Takahashi, 1996), the inferior colliculus (IC) of the rabbit (Fitzpatrick et al., 1995) and the IC of the cat (Litovsky, 1998; Litovsky and Yin, 1998; Litovsky and Delgutte, 2002; Yin, 1994) and revealed that correlates of the precedence effect already exist on the level of the IC. Response rates of single neurons depend similarly on the direction of lead and lag sources and the lead-lag delay as the perceived location of the compound stimulus (lead and lag) in corresponding behavioral tasks performed by humans or cats (see, e.g., Tollin and Yin, 2003). Fitzpatrick et al. found evidence that suggests a progressive increase of the suppressive effect of the leading stimulus along the ascending auditory pathway. Furthermore, results indicate that the precedence effect is not exclusively based on binaural cues, but is also observable in the sagittal plane (Litovsky et al., 1997).

Commonly, researchers used double click-pairs providing directional information and recorded responses from single neurons. Generally, recovery curves (response to the lag as a function of the lead-lag delay) show a huge variability for different cells. For single neurons of the IC in the cat, Yin (1994) found a median lead-lag delay of $20 \mathrm{~ms}$ for a $50 \%$ recovery. Similar results were obtained by Litovsky et al. (2001) and Litovsky and Delgutte (2002) (lead-lag delay $32 \mathrm{~ms}$ ). Fitzpatrick et al. (1999) found increasing lead-lag delays for a $50 \%$ recovery in structures of the ascending auditory pathway (from the auditory nerve up to the cortex) in cats and rabbits. They obtained recovery times of about $2 \mathrm{~ms}$ for the early stages like the auditory nerve (cat), the anteroventral cochlear nucleus (cat) and the superior olivary complex (rabbit). In neurons of the IC (rabbit) recovery times average around $7 \mathrm{~ms}$, and for neurons of the auditory cortex (rabbit) recovery times of about $20 \mathrm{~ms}$ were determined.

As explained by Fitzpatrick et al. (1999), the increasing recovery times for ascending stages of the auditory pathway may not be directly linked to the precedence effect, because the duration of the suppression clearly exceeds the echo threshold. They assume that the precedence effect is absent for lead-lag delays longer than the echo threshold. Moreover, no systematic differences between the recovery times obtained from monau- ral and binaural stimulation were found (Fitzpatrick et al., 1999). Therefore, the analysis of recovery curves may not reveal strong evidence about the site of the generation of the precedence effect.

However, a more recent study (Litovsky and Delgutte, 2002) revealed that neural correlates of the precedence effect are highly related to localization cues. Applying a virtual space technique, they found that, for a majority of IC neurons, the excitation produced by the lead as well as the suppression of the lag response strongly depended on directional cues. However, in several physiological studies researchers also encountered many neurons whose responses to the lag were not suppressed due to the presence of the lead and whose characteristics were uncorrelated and therefore unpredictable from the response to the lead (see, e.g., Litovsky et al., 2001). These findings argue against a complete monaural suppression effect like forward masking because not all responses from all neurons were suppressed. This may be interpreted as a specific information suppression, i.e., some information is suppressed and other information is passed to higher levels of the auditory pathway.

Although the precedence effect in humans was intensively studied, it is still unknown whether it is a result of peripheral or central processes. Blauert (1997, p. 420) describes the precedence effect as 'the result of evaluation and decision processes in higher stages of the nervous system during which, in addition to auditory cues, cues from other sensory modalities and prior knowledge are taken into consideration'. In his point of view the precedence effect is a top-down process, where peripheral processes play an important role. He points out that the central nervous system decides whether a cue is enhanced or suppressed and therefore controls the peripheral processing.

In contrast, Hartung and Trahiotis (2001) emphasize the importance of peripheral processes. They show that the precedence effect can to a great amount be explained by peripheral processes without any top-down processes. In short, they propose within-filter interactions of the peripheral auditory filters which argues for bottom-up processes in the precedence effect.

Liebenthal and Pratt $(1997,1999)$ recorded brainstem and middle-latency responses to click-pairs comprising a diotic lead and a dichotic lag stimulus (interaural time differences (ITDs) from 0 to $900 \mu$ s) with lead-lag delays from 3 to $20 \mathrm{~ms}$. The binaural peak amplitude in the echo-evoked middle-latency component $\mathrm{Pa}$ was maximally reduced for a lead-lag delay of $4 \mathrm{~ms}$. However, the suppression of the $\mathrm{Pa}$ depended less strongly on the lead-lag delay than their corresponding psychoacoustic position judgements. Liebenthal and Pratt did not find a consistent effect on any preceding components, i.e., no suppression effect was observed in auditory brainstem responses (ABRs). 
The current study uses electroencephalography (EEG) in order to gain knowledge about how the precedence effect is reflected in the successive auditory processing stages in the human brain. In contrast to the work of Liebenthal and Pratt, the present study focuses on ABR and cortical auditory evoked potentials (CAEPs). Hence, also several stages of the auditory pathway are investigated. Especially, the question is addressed whether the precedence effect results from a poor sensitivity of peripheral processing or from specific cognitive processes.

In situations where the precedence effect operates, the perception of a stimulus pair is unlike its physical features at the eardrums, i.e., two auditory stimuli are presented but a single fused event is perceived and, additionally, spatial information of the lagging stimulus is suppressed. Hence, the representation of the stimulus features in the ABRs would argue for a sufficient sensitivity of the peripheral processing which suggests that information about both stimuli is completely available on the early stages of the auditory pathway.

In contrast, the representation of the perceived auditory image in the ABR would indicate that the precedence effect is already operating on the early stages of the auditory pathway. The analysis of the ABR in this study focuses on the determination of amplitude and latency of the prominent wave $V$. Several studies suppose that this ABR component is mainly generated by the lateral lemniscus and its termination into the IC (see, e.g., Møller and Jannetta, 1982; Scherg and von Cramon, 1985; Møller et al., 1994).

In addition to the ABRs, MMN components of the CAEP were determined using stimuli that are known to evoke the precedence effect in behavioral tasks. The MMN component is believed to be produced by a process that compares the neuronal trace elicited by a frequent reference stimulus ('standard') with any new incoming auditory event ('deviant') that produces its own neuronal trace, i.e., an MMN component is produced if a significant difference between standard and deviant is perceived by the auditory system (Näätänen et al., 1978). Hence, MMN components can only be elicited if information related to the altered stimulus feature has at least partly been processed before.

Additionally, the current study comprises psychoacoustical measurements determining discrimination thresholds of the lag-ITD as a function of the lead-lag delay. Results were obtained from the same group of subjects as for the EEG recordings. In doing so, amplitudes and latencies of the MMN components can be related to the psychoacoustically obtained discrimination thresholds. As the generation of the MMN components is assumed to be dependent on the perception of the stimulus, MMN components recorded here are expected to reflect the results of the psychoacoustical measurements, i.e., small $\mathrm{MMN}$ amplitudes are expected for lead-lag delays where high discrimination thresholds are obtained and vice versa. An agreement between psychoacoustical and neurophysiological data would indicate that information related to the precedence effect is at least partially processed prior to the stage of the generation of the MMN. Additionally, this would show that the precedence effect is still effective on the level of the MMN.

\section{Methods}

\subsection{Auditory evoked potentials}

\subsubsection{Subjects}

A total of eight subjects (one female, seven male), aged from 26 to 40, mean 31, participated in either the ABR or CAEP recordings. They were normal hearing according to their subjective assessment and their audiogram (hearing loss $<20 \mathrm{~dB}$ between 0.125 and $8 \mathrm{kHz}$ ). They all participated in previous EEG experiments and were therefore familiar with the recording procedure. Five subjects took part in the ABR experiments. During the recordings they lay in a darkened booth and were asked to relax. Some of them even managed to sleep as all stimuli were presented at a level of $40 \mathrm{~dB}$ nHL (normal hearing level). Eight subjects participated in the CAEP recordings. During these recordings subjects sat in a reclining chair and watched a selfselected subtitled movie. All except one subject that participated in the ABR recordings also participated in the CAEP recordings.

\subsubsection{Apparatus}

Basically, the setup for the acquisition of both ABRs and CAEPs are identical. Stimuli were generated digitally on a DSP32C card and were DA-converted at a sampling frequency of $50 \mathrm{kHz}$. Signals were presented to the subjects by insert-ear-phones (Etymotic Research ER-2). The EEG was recorded from 3 (ABR) or 31 (CAEP) positions of the scalp, respectively. All electrodes were referenced to $\mathrm{CZ}$. Additionally, in the CAEP experiments the horizontal and vertical EOG (electrooculogram) were recorded by bipolar electrodes.

Recorded signals were pre-amplified inside the electrically and acoustically shielded booth by a factor of 150 . Outside the booth the signals passed a DC-coupled differential amplifier where they were further amplified by a factor of 33.3 yielding a total amplification of $74 \mathrm{~dB}$. Raw data were A/D-converted with a resolution of 16 bits and stored continuously to disc. The sampling frequency was $10 \mathrm{kHz}$ for the $\mathrm{ABR}$ recordings and $1 \mathrm{kHz}$ for the CAEP recordings. The artifact level for all recordings was set to $\pm 500 \mu \mathrm{V}$. Epoching, filtering, artefact rejection, sorting and averaging of the data was done offline. 


\subsubsection{Stimuli}

Auditory brainstem responses. Single clicks for the ABR experiments had a duration of $60 \mu$ s (3 samples). Click-pairs with six different lead-lag delays and two lag-ITDs, respectively, were presented within sequences in random order. Each sequence contained 10,000 stimuli. As, on average, the interstimulus interval was $70 \mathrm{~ms}$ (a jitter of $10 \mathrm{~ms}$ was employed), each sequence had a duration of about $12 \mathrm{~min}$. For each stimulus condition 10,000 responses were collected.

Cortical auditory evoked potentials. Two types of stimuli, reference and deviant stimuli, were applied (see Fig. 1). Both stimuli consisted of a lead and a lag click. The lead click of both stimuli as well as the lag click of the reference had an ITD of zero. Deviants had an ITD of $800 \mu \mathrm{s}$ in the right channel of the lag. This yields a lateralized perception to the left hand side. Like in the ABR recordings each single click had a duration of $60 \mu \mathrm{s}$ (3 samples). Lead-lag delay values of 1, 5 and $20 \mathrm{~ms}$ were applied.

\subsubsection{Recordings}

Auditory brainstem responses. Auditory brainstem responses were recorded in two different experiments: in Experiment I ABRs were recorded using diotic double click-pairs with lead-lag delays from 0 to $5 \mathrm{~ms}$ in steps of $1 \mathrm{~ms}$. In Experiment II ABRs were recorded using diotic (lag-ITD $=0 \mu \mathrm{s}$ ) as well as dichotic (lagITD $=300 \mu \mathrm{s}$ ) double click-pairs for lead-lag delays of 0,5 and $20 \mathrm{~ms}$.

Cortical auditory evoked potentials. Cortical auditory evoked potentials were collected according to the paradigm of the mismatch negativity. Therefore, throughout the CAEP recordings stimuli were presented within sequences with a stimulus repetition rate of $2 \mathrm{~Hz}$. All stimuli within one sequence had the same lead-lag delay. Each sequence contained frequent (about $88 \%$ ) reference and rare (about 12\%) deviant stimuli which appeared at randomly chosen positions of the sequence on condition that at least three reference stimuli follow (precede) a deviant. Each sequence contained 2100 stimuli (1850 reference and 250 deviant stimuli) and had a duration of $17.5 \mathrm{~min}$. For each of the eight subjects, responses to 1000 deviants and 7400 references were collected for each lead-lag delay. The total net measurement time was $3.5 \mathrm{~h}$ per subject and was divided into two sessions.

\subsubsection{Data analysis}

Raw data of all experiments were cut into epochs with durations of $70 \mathrm{~ms}$ (ABR) or $500 \mathrm{~ms}$ (CAEP), respectively. Epochs were filtered with a recursive bandpass filter of second order and corner frequencies of 100 and $1500 \mathrm{~Hz}(\mathrm{ABR})$ or 1 and $20 \mathrm{~Hz}$ (CAEP), respectively. As a 'forward-backward' filter design was applied no dispersion due to different group delays occurred. After filtering, epochs obtained by identical stimuli were sorted and averaged. Artifacts were accounted for by an iterated weighted averaging technique, i.e., epochs were weighted with their inverse power (Riedel et al., 2001).

For both ABR experiments amplitudes and latencies of the first prominent wave $V\left(V_{\mathrm{a}}\right)$ for each lead-lag delay were obtained by determining the maximum voltage values in the time interval from 6.5 to $7.5 \mathrm{~ms}$ after stimulus onset. This was a simple and adequate method as all peaks fell within this time interval.

For the second wave $V\left(V_{\mathrm{b}}\right)$ the lookup interval was shifted in latency according to the lead-lag delay. In order to gain accuracy especially in the latency discrimination, ABR data were upsampled by a factor of 10 , i.e., the sampling frequency was changed from 10 to $100 \mathrm{kHz}$.

In the CAEP experiments data was rereferenced to NZ (nose) in order to facilitate comparisons with data
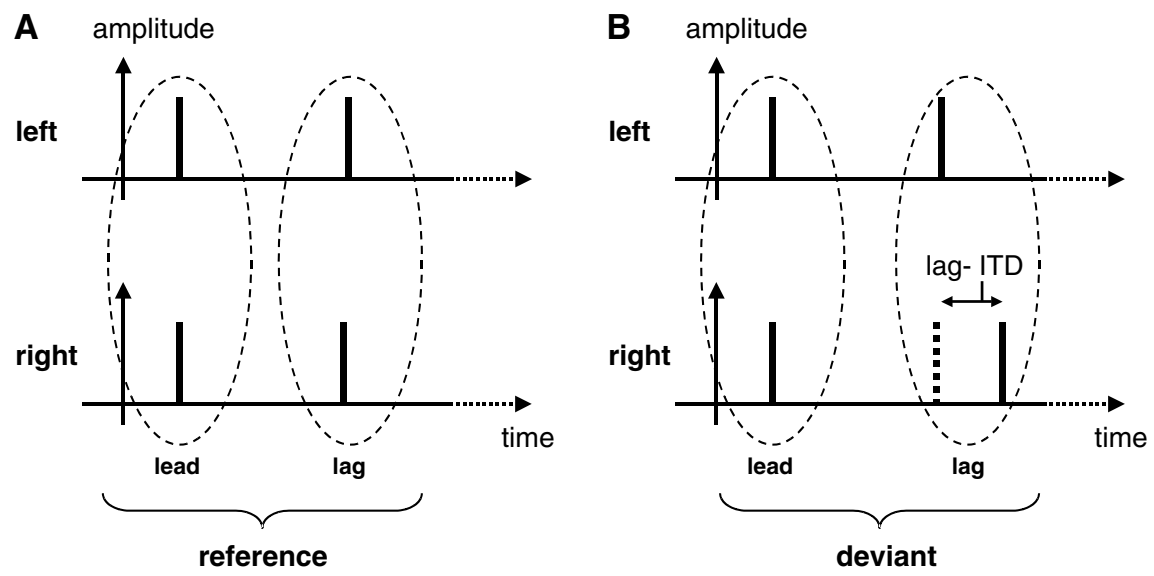

Fig. 1. Sketch of reference and deviant stimuli that were used for the CAEP recordings. Each stimulus consists of two click-pairs, lead and lag. (A) The diotic reference stimulus. (B) The dichotic deviant stimulus with a lag-ITD of $800 \mu \mathrm{s}$. 
of other studies (see, e.g., Schröger, 1996). Using the nose as a reference, the MMN component changes its polarity from frontal to parietal electrodes and is therefore identified more easily. However, as in our pilot studies the signal-to-noise ratio (SNR) was much worse with the nose instead of $\mathrm{CZ}$ as a reference we decided to collect the data at best possible SNR and to rereference the data offline. Difference waveforms were obtained by subtracting the response to reference stimuli from the response to deviant stimuli. Latencies of the MMN components were detected by determining the largest peak of each difference waveform. MMN components were considered significant if the peak voltage exceeded the standard error of the average waveform (see also Riedel and Kollmeier, 2002). The standard error of the difference curves was determined according to equation $\sigma_{\text {diff }}=\sqrt{\sigma_{\text {std }}^{2}+\sigma_{\text {dev }}^{2}}$.

\subsection{Psychoacoustics}

The psychoacoustical experiments determined the individual discrimination threshold of a lag-ITD, i.e., the discrimination threshold of an ITD in the presence of a preceding diotic click (the lead).

\subsubsection{Subjects}

All subjects that participated in the CAEP recordings also took part in the psychophysical measurements. Three of the subjects had intensive experience in psychoacoustical measurement tasks. The remaining five had only little or no prior experience in psychoacoustical measurement tasks but had several practice runs until the actual data collection began. In particular, attention was paid to the subjects' vigilance. Most subjects performed about five measurements in a row (net measuring time about $20 \mathrm{~min}$ ) until they reported fatigue. Measurements either continued after a longer break or on the next day.

\subsubsection{Apparatus}

The psychophysical experiments were performed in a double-walled sound proof booth (IAC 1203A). Signal generation and presentation were controlled by a personal computer. Stimuli were generated digitally at a sampling rate of $96 \mathrm{kHz}$, transformed by an D/A converter (type SEK'D 2496 DSP) amplified by a preamplifier (type Behringer HA 4400) and presented via headphones (type AKG K 501) at a level of $40 \mathrm{~dB}$ nHL (normal hearing level). The subjects sat in front of the monitor of the personal computer and gave their responses by pressing predetermined buttons on the keyboard.

\subsubsection{Stimuli}

Stimuli employed in the psychoacoustic tasks were basically identical to those applied in the CAEP record- ings (see Fig. 1): the basic component of the stimuli was a pulse of about $52 \mu \mathrm{s}$ in duration (five samples with a value of one at a sampling frequency of $96 \mathrm{kHz}$ ). The acoustic pulse was therefore approximately the impulse response of the earphones (AKG 501). The measured impulse response of the headphones had a duration of about $1 \mathrm{~ms}$.

\subsubsection{Measurements}

In order to improve the significance of the comparison between the results of the psychophysical and the EEG measurements, the stimulus presentation mode of the psychophysical measurements was adapted to that of the EEG measurements. Stimulus sequences contained about 210 stimuli (30 deviants and about 180 reference stimuli) and were presented at a repetition rate of $2 \mathrm{~Hz}$ (duration about $2 \mathrm{~min}$ ).

Deviants occurred on randomly chosen positions of the sequence on condition that at least three reference stimuli precede (follow) a deviant. Deviants had one of the fixed lag-ITDs from 150 up to $900 \mu$ s in steps of $150 \mu$ s. Each of the six different lag-ITDs was presented 5 times in each sequence in interleaved order. The lead-lag delay was varied as a parameter over the sequences but was constant within each sequence. Eight lead-lag delays $(0,1,2,3,5,7,10$ and $20 \mathrm{~ms})$ were applied so that a total of 48 different sequences (6 sequences per lead-lag delay) were presented (see Fig. 2).

Subjects were asked to hit a button on the keyboard as fast as possible after they detected a deviant stimulus. A subjects' response was treated as a hit if any button on

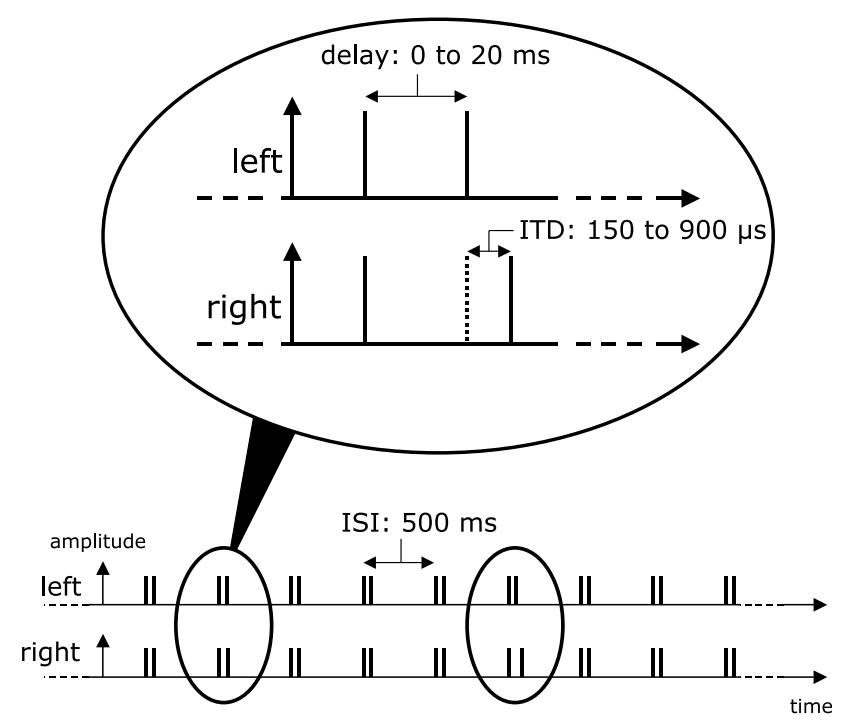

Fig. 2. Sketch of the stimulus sequence used in the psychoacoustical measurements. The sequence consisted of about 210 lead-lag clickpairs ( 30 deviants and about 180 reference stimuli). The ITD of the lag click of the deviant was varied from 150 up to $900 \mu$ s in steps of $150 \mu$ s. 
the keyboard was pressed within one second after the presentation of the deviant. In doing so, the attention was turned strongly to the stimulus sequence. The subjects received no feedback during the measurement.

An analysis of the key-press statistics shows that subjects did not have difficulties with the task: About $87 \%$ of the key-presses were given within $0.5 \mathrm{~s}$ after the presentation of the deviant, less than $10 \%$ within $0.5 \mathrm{~s}$ and $1 \mathrm{~s}$ and less than $3 \%$ of the key-presses were given with a delay larger than $1 \mathrm{~s}$. The latter key-presses were treated as false alarms.

A psychometric function according to equation $f\left(\operatorname{ITD}_{\text {lag }}\right)=\left(1+\mathrm{e}^{\left(a+b \cdot \operatorname{ITD}_{\text {lag }}\right)}\right)^{-1}$ was fitted to the individual data set where $a$ determines the horizontal shift and $b$ the slope of the function. The discrimination threshold was determined as the lag-ITD that corresponds to a $70.7 \%$ correct performance. For one subject, the discrimination thresholds of the lag-ITD were far beyond $900 \mu \mathrm{s}$, the largest lag-ITD employed. Discrimination thresholds for this subject were obtained in a supplementary measurement using the same procedure but applying larger lag-ITD values (up to $1200 \mu \mathrm{s}$ ).

\section{Results}

\subsection{ABR recordings}

Fig. 3 shows ABRs of Experiment I for subject 1. Fig. $3 \mathrm{~A}$ shows sketches of the diotic stimuli which differ in lead-lag delay. In Fig. 3B corresponding ABRs of the three measurement channels (A1, A2 and IZ) are depicted. For a lead-lag delay of $0 \mathrm{~ms}$ the typical characteristics of an ABR for a transient stimulus are observable. Its most prominent component is wave $V$ $\left(V_{\mathrm{a}}\right)$ at a latency of about $7 \mathrm{~ms}$. For lead-lag delays larger than $1 \mathrm{~ms}$ a second wave $V\left(V_{\mathrm{b}}\right)$ emerges.

In Fig. 4 differences in latency (A) and amplitude (B) between this emerging wave $V_{\mathrm{b}}$ and the first wave $V_{\mathrm{a}}$ averaged across channels and subjects are shown. The latency difference between the second and first wave $V$ $\left(t_{V_{\mathrm{b}}}-t_{V_{\mathrm{a}}}\right)$ increases linearly with the lead-lag delay of the stimulus. However, all latency differences are about $0.4 \mathrm{~ms}$ larger than the lead-lag delay of the stimulus. Interindividual standard deviations of the latency differences decrease with increasing lead-lag delays.

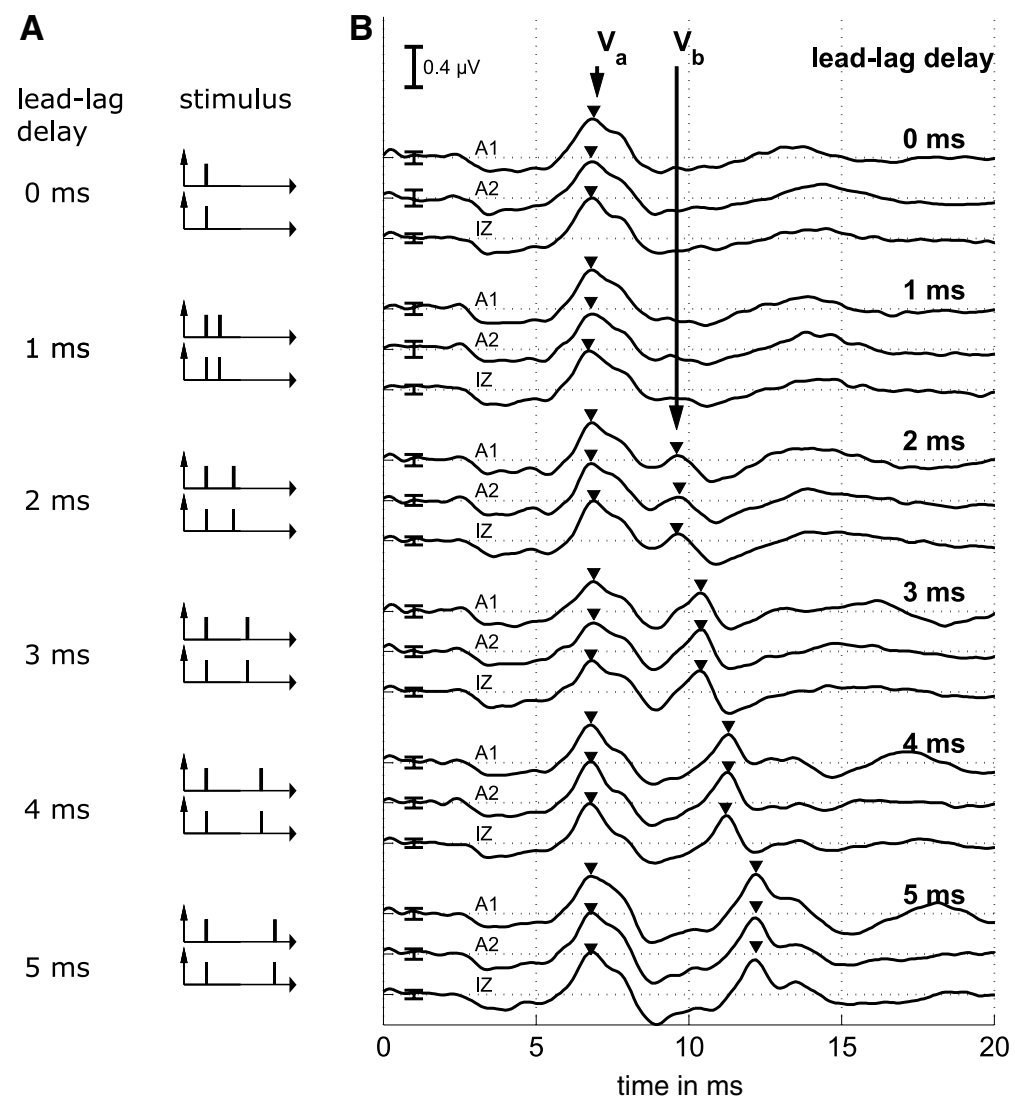

Fig. 3. (A) Sketch of the time signals used for the ABR recordings in Experiment I, diotic stimulation with increasing lead-lag delay (0-5 ms), presentation level $40 \mathrm{~dB}$ nHL. (B) Corresponding ABRs of subject 1. Potentials of three channels (A1, A2 and IZ) are shown for each lead-lag delay. Errorbars at a latency of $1 \mathrm{~ms}$ indicate the fivefold intraindividual standard error $( \pm 5 \sigma)$. 

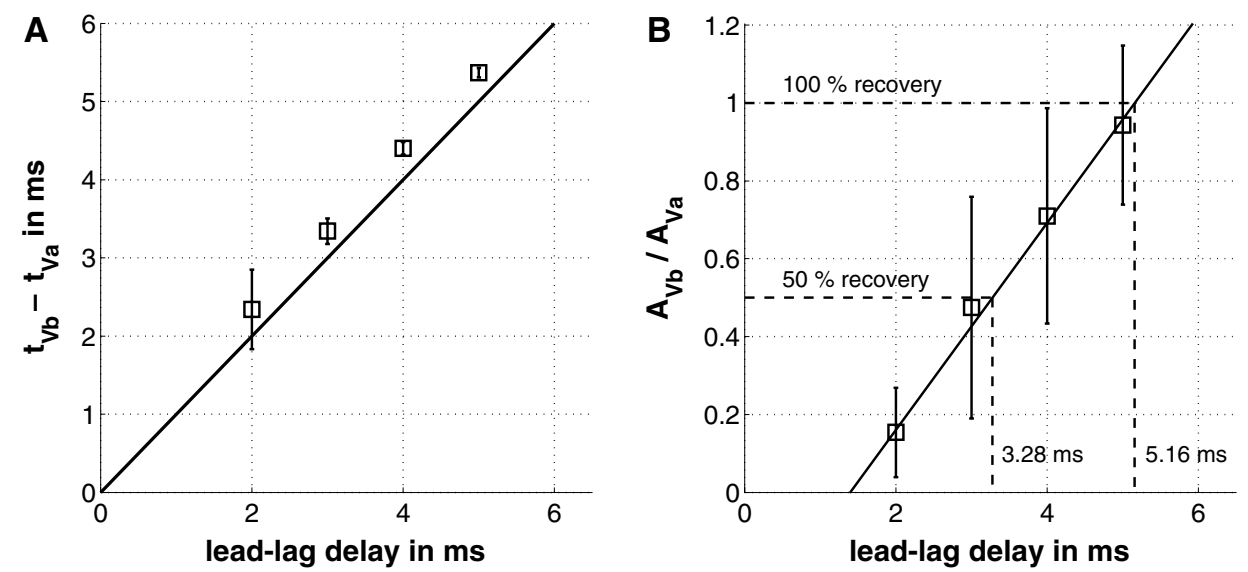

Fig. 4. (A) Latency differences between the second and first wave $V\left(t_{V_{\mathrm{b}}}-t_{V_{\mathrm{a}}}\right)$ of the ABRs obtained in Experiment I as a function of the lead-lag delay. (B) Amplitude ratio between the second and first wave $V\left(A_{V_{\mathrm{b}}} / A_{V_{\mathrm{a}}}\right)$ as a function of the lead-lag delay. The line is the result of a linear fit of the amplitude ratio values. The $50 \%$ and $100 \%$ recovery values were taken from the fit. Both plots show mean values averaged over all subjects and channels with interindividual standard deviations.

Fig. 4B shows the amplitude ratio of the second and first wave $V,\left(A_{V_{\mathrm{b}}} / A_{V_{\mathrm{a}}}\right)$. The amplitudes of wave $V_{b}$ increase fairly linear with increasing lead-lag delays up to the maximum lead-lag delay of $5 \mathrm{~ms}$ applied. After averaging across channels and subjects for each leadlag delay, all values were normalized to the mean amplitude value of the first wave $V$. The amplitude of wave $V_{\mathrm{b}}$ at a lead-lag delay of $5 \mathrm{~ms}$ is similar to that of wave $V_{\mathrm{a}}$. A linear function was fitted to the amplitudes of wave $V_{\mathrm{b}}$ using a $\chi^{2}$-fit, i.e., by weighting the four amplitudes by their corresponding interindividual standard devia- tion. The lead-lag delays corresponding to a $50 \%$ and a $100 \%$ recovery of wave $V_{\mathrm{b}}$ determined by the fit were 3.28 and $5.16 \mathrm{~ms}$, respectively.

Results obtained from Experiment II are depicted in Fig. 5 and Table 1. Fig. 5A outlines the stimulus signals that were applied in order to obtain the respective ABRs shown in Fig. 5B. Rows 1, 3 and 5 show results of the diotic stimuli, i.e., neither lead nor lag had an ITD. In contrast, in rows 2,4 and 6 , the right channel of the lag stimulus was delayed by an amount of $300 \mu$ s yielding a dichotic stimulation. Potentials recorded from

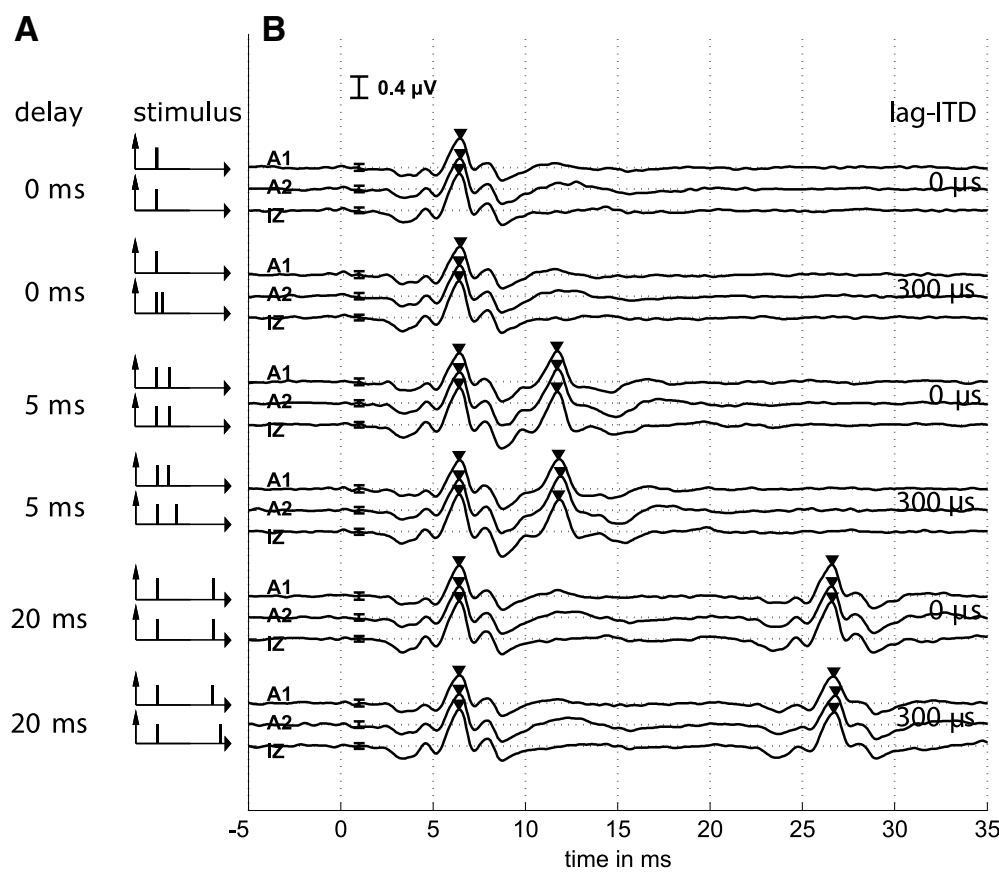

Fig. 5. (A) Time signals of Experiment II. Diotic and dichotic double click-pairs with lead-lag delays of 0, 5 and 20 ms were presented. Dichotic stimuli had a lag-ITD of $300 \mu$ s. (B) Auditory brainstem responses of subject 2 from Experiment II. Data were collected from three channels (A1, A2 and IZ). Errorbars at a latency of $1 \mathrm{~ms}$ show the fivefold intraindividual standard errors $( \pm 5 \sigma)$. 
Table 1

Latency differences $\left(t_{V_{\mathrm{b}}}-t_{V_{\mathrm{a}}}\right)$ between waves $V_{\mathrm{b}}$ and $V_{\mathrm{a}}$ for diotic $($ ITD $=0 \mu \mathrm{s})$ and dichotic $($ ITD $=300 \mu \mathrm{s})$ stimulation from Experiment II

\begin{tabular}{lll}
\hline$\left(t_{V_{\mathrm{b}}}-t_{V_{\mathrm{a}}}\right)$ for Experiment II & \multicolumn{2}{l}{ Lead-lag delay } \\
\cline { 2 - 3 } & $5 \mathrm{~ms}$ & $20 \mathrm{~ms}$ \\
\hline ITD $=0 \mu \mathrm{s}$ & $5.30 \pm 0.08 \mathrm{~ms}$ & $20.11 \pm 0.03 \mathrm{~ms}$ \\
ITD $=300 \mu \mathrm{s}$ & $5.41 \pm 0.12 \mathrm{~ms}$ & $20.26 \pm 0.03 \mathrm{~ms}$ \\
\hline
\end{tabular}
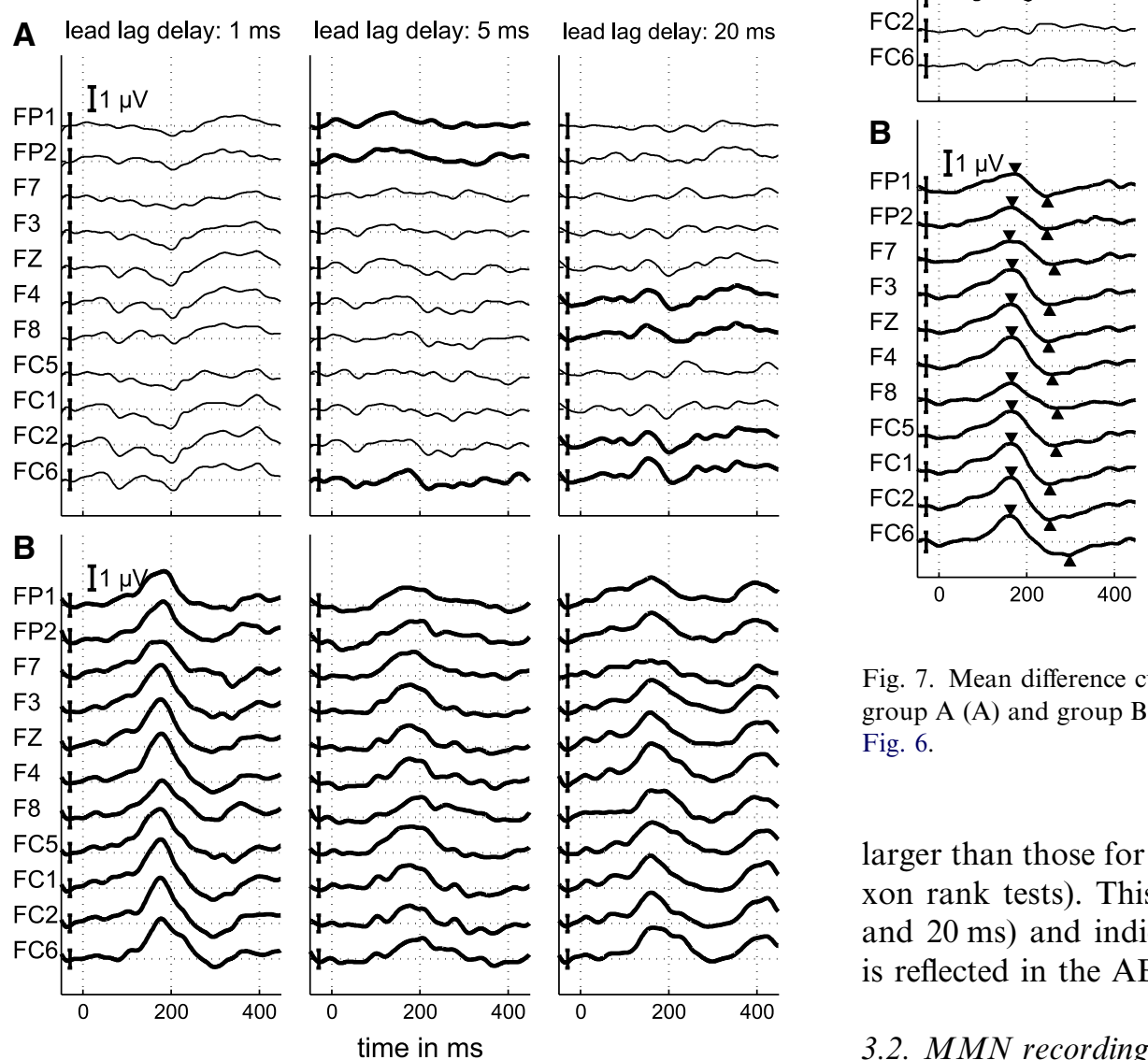

Fig. 6. (A) Difference curves (response to deviant minus response to reference stimulus) of the frontal channels for lead-lag delays of 1,5 and $20 \mathrm{~ms}$ of subject 6 rereferenced to NZ. Errorbars at a latency of $-30 \mathrm{~ms}$ in each channel show the intraindividual standard error. Thin lines indicate channels in which the MMN component is smaller than the respective intraindividual standard error. (B) Difference curves of subject 2. All channels show significant MMN components (thick lines)

three channels (A1, A2 and IZ) for subject 2 are shown. Amplitudes of all wave $V$ s are nearly identical. According to Wilcoxon rank tests no significant differences were found for the amplitudes across different lag-ITDs or the two lead-lag delays. Overall, for lead-lag delays of 5 and $20 \mathrm{~ms}$, latency differences between the first $\left(V_{\mathrm{a}}\right)$ and second $\left(V_{\mathrm{b}}\right)$ wave $V$ are slightly larger than the lead-lag delay of the stimulus (see Table 1). On average, latency differences for dichotic stimulation are slightly
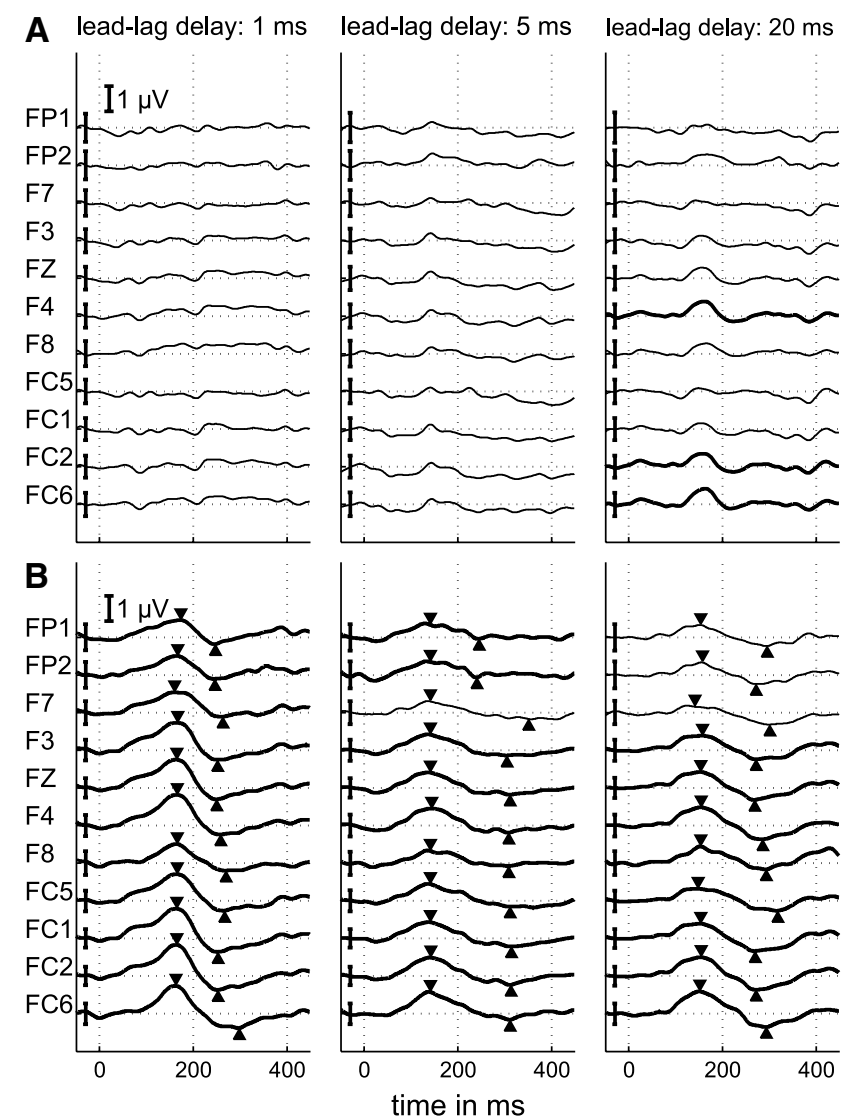

Fig. 7. Mean difference curves of the frontal channels for subjects of group A (A) and group B (B). The layout of the plot is the same as in Fig. 6.

larger than those for diotic stimulation $(p<0.05$, Wilcoxon rank tests). This holds for both lead-lag delays (5 and $20 \mathrm{~ms}$ ) and indicates that the ITD of the stimulus is reflected in the ABRs.

\section{2. $M M N$ recordings}

Fig. 6A shows difference curves of the frontal channels rereferenced to a nose electrode (NZ) of subject 6 for lead-lag delays of 1, 5 and $20 \mathrm{~ms}$. As positive voltage values are plotted downwards, MMN components are directed upwards. For lead-lag delays of 1 and $20 \mathrm{~ms}$ subject 6 shows significant MMN peaks (peak amplitude $>1 \sigma$ ) only in six and five out of eleven channels (thick lines). In none of the channels a significant MMN-peak could be detected at a lead-lag delay of $5 \mathrm{~ms}$ (thin lines). Difference curves of subject 2 are plotted in Fig. 6B. For the three lead-lag delays peak amplitudes are larger than the intraindividual standard error in all channels. They peak at a latency of about $180 \mathrm{~ms}$.

Subjects that participated in the CAEP recordings could be divided into two groups according to their potentials. If less than $2 / 3$ of the frontal channels for lead-lag delays of 1 and $20 \mathrm{~ms}$, i.e., 15 out of 22 chan- 


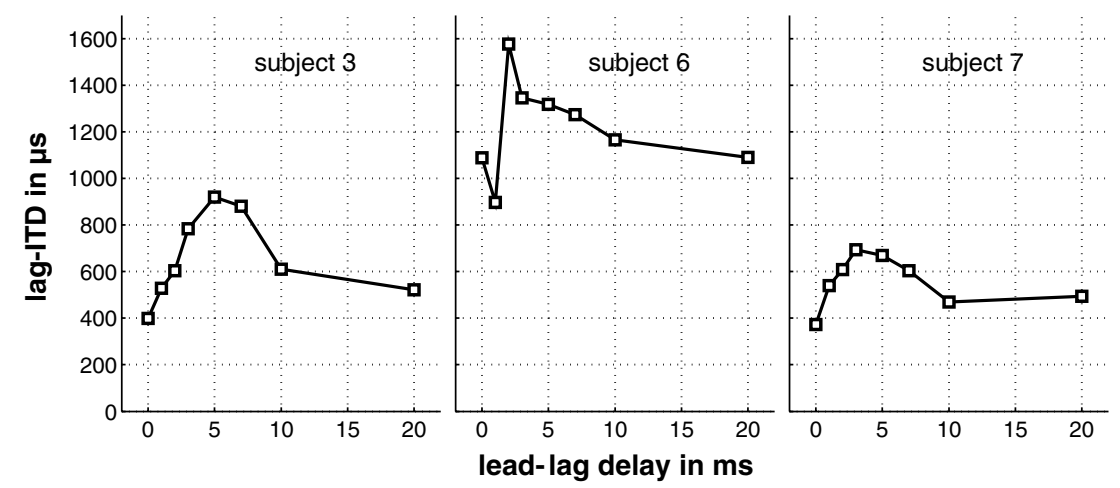

Fig. 8. Discrimination thresholds of the three subjects that were assigned to group A according to their MMN amplitudes. Lag-ITD values that yield $70.7 \%$ correct performance are plotted over the lead-lag delay from 0 to $20 \mathrm{~ms}$.

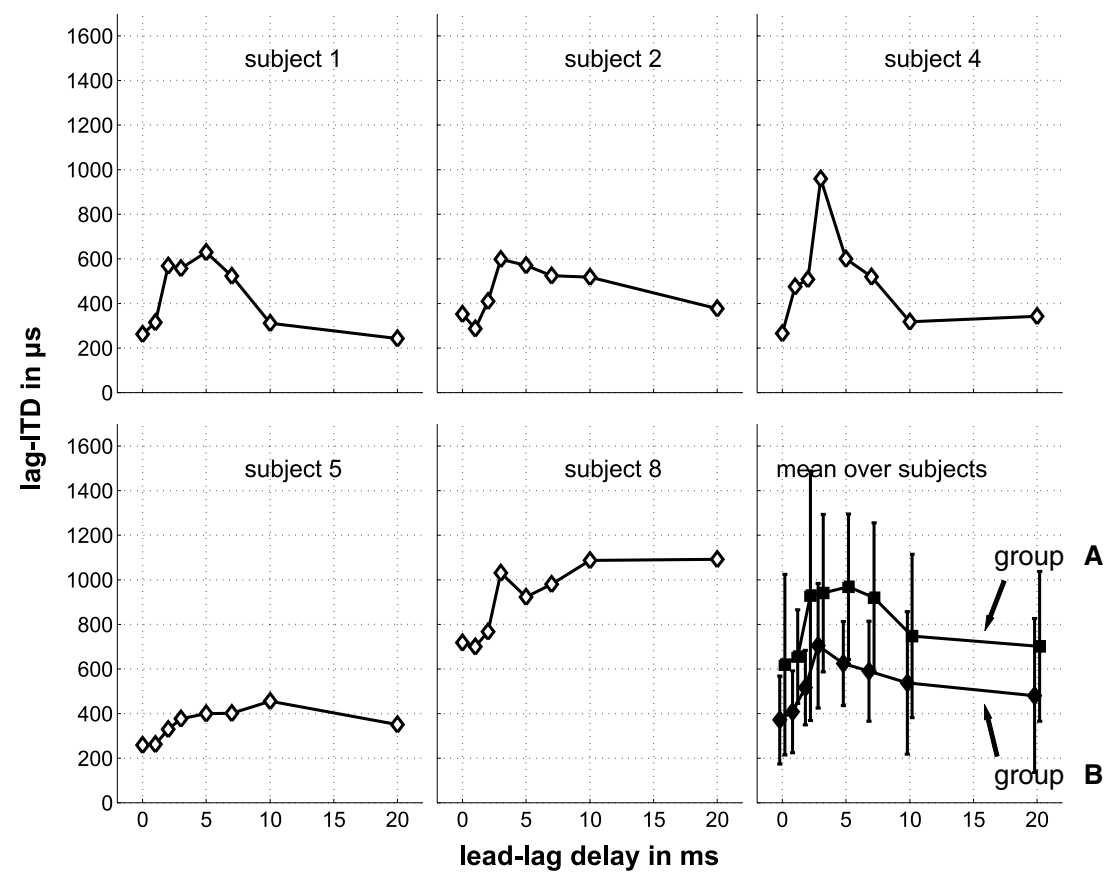

Fig. 9. Discrimination thresholds of the five subjects that were assigned to group B according to their MMN amplitudes. Lag-ITD values that yield $70.7 \%$ correct performance are plotted over the lead-lag delay from 0 to $20 \mathrm{~ms}$. The lower right panel shows average values of both groups as well as interindividual standard deviations.

Table 2

Comparison between the psychoacoustical and the electrophysiological performance of the five subjects of group $\mathrm{B}$, mean $\pm 1 \sigma$

\begin{tabular}{llll}
\hline & \multicolumn{2}{l}{ Lead-lag delay } \\
\cline { 2 - 4 } & $1 \mathrm{~ms}$ & $5 \mathrm{~ms}$ & $20 \mathrm{~ms}$ \\
\hline MMN amplitude $(\mu \mathrm{V})$ & $1.38 \pm 0.34$ & $0.85 \pm 0.18$ & $1.03 \pm 0.22$ \\
Amplitude ratio $(\%)$ & 100 & 62 & 75 \\
Discrimination threshold $(\mu \mathrm{s})$ & $407 \pm 183$ & $624 \pm 189$ & $481 \pm 345$ \\
Threshold ratio $(\%)$ & 100 & 153 & 118 \\
\hline
\end{tabular}

nels (11 frontal channels for a lead-lag delay of $1 \mathrm{~ms}$ and 11 frontal channels for a lead-lag delay of $20 \mathrm{~ms}$ ), showed significant MMN components, subjects were assigned to group A (3 subjects), the other to group B (5 subjects).
Fig. 7 shows difference waveforms averaged across the three subjects of group A (A) and the five subjects of group B (B), respectively. The layout of the plot is identical to that of Fig. 6. Again, channels in which the MMN peak exceeds the corresponding standard error are indicated with thick lines. In group A waveforms, significant MMN components only appear in three channels at a lead-lag delay of $20 \mathrm{~ms}$, whereas in group B waveforms, all except four channels show a significant MMN component. For group B waveforms, MMN peaks as well as the following minima are marked with triangles. Latencies of the maxima and peakto-peak values between maxima and minima were determined. According to Wilcoxon rank tests MMN peak-to-peak values for a lead-lag delay of $5 \mathrm{~ms}$ 


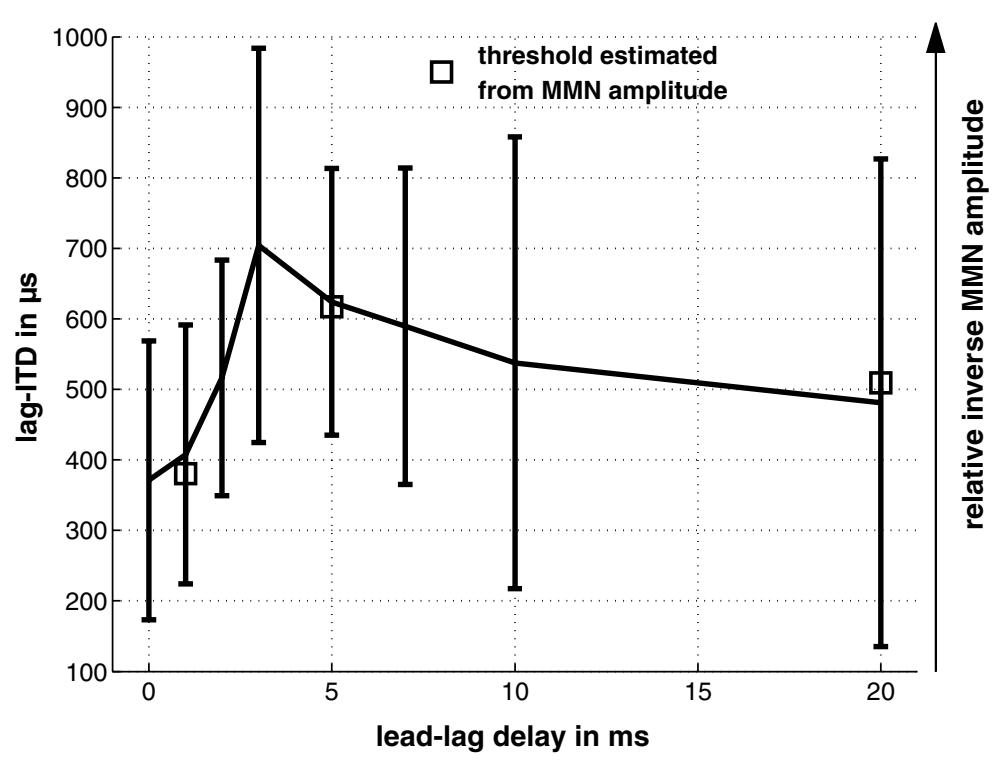

Fig. 10. Comparison between the psychoacoustical discrimination performance and the corresponding relative inverse amplitude of the MMN component. Both data sets were averaged across the five subjects of group B. Lag-ITDs that yielded 70.7\% correct performance in the discrimination task are plotted for lead-lag delays from 0 to $20 \mathrm{~ms}$. Errorbars show interindividual standard deviations. Squares indicate the predicted discrimination threshold by analyzing the MMN amplitude.

$(0.85 \pm 0.18 \mu \mathrm{V})$ are significantly smaller than those for lead-lag delays of $1 \mathrm{~ms}(1.38 \pm 0.34 \mu \mathrm{V})$ and $20 \mathrm{~ms}$ $(1.03 \pm 0.22 \mu \mathrm{V})$, respectively. Additionally, MMN latencies are significantly smaller for a lead-lag delay of $5 \mathrm{~ms}(138 \pm 4 \mathrm{~ms})$ in comparison to MMN latencies for lead-lag delays of $1 \mathrm{~ms}(166 \pm 4 \mathrm{~ms})$ and $20 \mathrm{~ms}$ $(152 \pm 9 \mathrm{~ms})$. Thus, smaller MMN amplitudes correspond to smaller peak latencies.

\subsection{Psychoacoustics}

Figs. 8 and 9 show discrimination thresholds obtained by the psychoacoustical experiments. Fig. 8 displays discrimination thresholds of the three subjects of group A, Fig. 9 shows discrimination thresholds of the five subjects that were assigned to group B, respectively. The lower right column of Fig. 9 shows mean values of group A (squares) and group B (diamonds) as well as the respective interindividual standard deviations.

Individual thresholds of both groups show a large variability. In contrast, averaged discrimination thresholds of both groups are similarly dependent on the lead-lag delay, i.e., thresholds are maximal for leadlag delays of about 3-5 ms and decrease for lower and higher lead-lag delays. However, lag-ITD values of group B are on the average about $280 \mu$ s lower than those of group A. Highest discrimination thresholds of about $950 \mu$ s (group A) and $700 \mu$ s (group B) were found for lead-lag delays around $3 \mathrm{~ms}$ whereas smallest discrimination thresholds of about $620 \mu$ s (group A) and $370 \mu$ s (group B) were found for a lead-lag delay of $0 \mathrm{~ms}$.

\subsection{Relation between MMN components and performance in discrimination tasks}

Difference curves averaged over the three subjects of group A are displayed in Fig. 7A. Psychoacoustical performances of subjects of group A for lead-lag delays of 1,5 and $20 \mathrm{~ms}$ are comparatively poor with discrimination threshold values of 655, 969 and $719 \mu \mathrm{s}$, respectively. As these lag-ITD values are close or even above the value of $800 \mu$ s that was used for the CAEP recordings, hardly any MMN component was elicited in these subjects.

The MMN components of the five subjects of group $\mathrm{B}$ are dependent on the lead-lag delay. On average (see Fig. 7B), amplitudes and latencies of the MMN components obtained with a lead-lag delay of $5 \mathrm{~ms}$ are significantly smaller compared to those MMN components obtained with lead-lag delays of 1 and $20 \mathrm{~ms}$.

Table 2 shows both peak-to-peak amplitudes of the MMN components and discrimination thresholds obtained from the psychoacoustical procedure averaged across channels and the five subjects of group B. Additionally, ratios among the MMN amplitudes and among the discrimination thresholds are specified, respectively.

Results show that the peak-to-peak amplitude of the MMN component decreases if the lead-lag delay changes from 1 to $5 \mathrm{~ms}$. At the same time the discrimination threshold increases, i.e., higher discrimination thresholds lead to smaller MMN peak-to-peak amplitudes. The further increase of the lead-lag delay from 5 to $20 \mathrm{~ms}$ yields a lower discrimination threshold again 
while the peak-to-peak amplitude of the MMN increases. Table 2 shows that discrimination thresholds increase/decrease to almost the same extent as the peak-to-peak amplitudes of the MMN components decrease/increase.

Fig. 10 compares the absolute discrimination threshold values as obtained from the psychoacoustical measurements with predicted discrimination thresholds according to the inverse ratio of the peak-to-peak amplitudes of the MMN components. Both data sets were obtained by averaging the results across the five subjects of group B that took part in either measurement. The solid line in Fig. 10 shows the discrimination threshold as a function of the lead-lag delay. As individual discrimination performance differs considerably (especially subject 8, see Fig. 9), comparatively large interindividual standard deviations appear, e.g., above $300 \mu$ s at a leadlag delay of $20 \mathrm{~ms}$ (without subject 8 interindividual standard deviations average around $100 \mu \mathrm{s}$ ). On average, the lag-ITD that subjects required to achieve a $70.7 \%$ correct performance in the psychoacoustical tasks was far below the lag-ITD value of $800 \mu$ s which was employed for the deviants in the CAEP recordings for all lead-lag delays. This indicates that the conditions for the CAEPs were all above detection threshold. Hence, a comparison between MMN data and psychophysiological data is achievable in this group of subjects (group B).

The estimate of absolute discrimination thresholds from peak-to-peak amplitudes of the recorded MMN components was done by scaling the inverted peakto-peak amplitude with a factor that provides the best fit to the empirical data as shown in Fig. 10. The scaling factor determined by a least-squares-fit was $525 \mu \mathrm{V} \mu \mathrm{s}$.

The estimated lag-ITD thresholds (diamonds in Fig. 10) for lead-lag delays of 1 and $5 \mathrm{~ms}$ lie slightly below the mean lag-ITD thresholds from the psychoacoustic measurements whereas the estimated value for a leadlag delay of $20 \mathrm{~ms}$ lies slightly above the average behavioral threshold.

\section{Discussion}

The general finding of this study is that the ABRs to double click-pairs follow closely the properties of the stimuli, i.e., the second click-pair elicits a second wave $V$ complex which can be predicted quite well from the response to single click-pairs. The CAEPs, on the other hand, seem to follow the perceptual impression of double click-pairs that exhibit a reduced response to the lag stimulus for lead-lag delays in order of $5 \mathrm{~ms}$. The different behavior of both types of electrophysiological recordings for the stimuli employed here will be discussed below.

\subsection{Relation between ABRs and the precedence effect}

\subsubsection{Experiment I}

In Experiment $\mathrm{I}$ of the ABR recordings diotic double click-pairs were presented with interclick intervals from 0 to $5 \mathrm{~ms}$. For lead-lag delays larger than $1 \mathrm{~ms}$ a second wave $V$ emerges which gradually increases in amplitude with increasing lead-lag delay. For a delay of $5 \mathrm{~ms}$ the amplitudes of the first and the second wave $V$ are almost identical.

Results of Experiment I can be related to previous studies. Some researchers recorded ABRs using maximum length sequences with short interclick intervals (see, e.g., Eysholdt and Schreiner, 1982; Burkard et al., 1990; Burkard, 1991). These studies show that at a level of $50 \mathrm{~dB}$ nHL with increasing interclick intervals from 1 to $10 \mathrm{~ms}$ amplitudes of wave $V$ increase from 0.2 to $0.35 \mu \mathrm{V}$ and latencies decrease from about 7.7 to $6.4 \mathrm{~ms}$. A similar result was observed in the present study, i.e., an increasing amplitude of the second wave $V$ with increasing lead-lag delay. For high repetition rates (small interclick intervals) the auditory system does not seem to be able to elicit equal potentials to every stimulus but exhibits a refractory state that extends to an interval of about $5 \mathrm{~ms}$. Additionally, Hey (2001) determined electrically evoked ABRs in cochlea implant patients using pulse-trains with interpulse intervals from 0.1 to $3.5 \mathrm{~ms}$. In two patients he found a full recovery of the second wave $V$ for an interpulse interval of about $3.5 \mathrm{~ms}$ which is $1.5 \mathrm{~ms}$ shorter than the lead-lag delay found for a full recovery in the experiments of the present study. However, for two other patients the amplitude of the second wave $V$ did not reach a full recovery for an interpulse interval of $3.5 \mathrm{~ms}$ (the largest interval applied). Extrapolating the dependency of the amplitude of the second wave $V$ on the interpulse interval as shown for both of these patients, a full recovery may be achieved for an interval of $4-5 \mathrm{~ms}$, a value quite similar to that obtained in the acoustically evoked ABRs of the current study.

Other parallels can be found in results of physiological studies: Parham et al. (1996) determined spike rates of auditory-nerve-fibers of cats while presenting double click-pairs with lead-lag delays of 1, 2, 4, 8 and $16 \mathrm{~ms}$. Results show a gradual recovery of the response to the second click which is comparable to the ABR findings of this study. A 50\% recovery was found for a lead-lag delay around $2.5 \mathrm{~ms}$ which corresponds well to the value of $3.28 \mathrm{~ms}$ for wave $V_{\mathrm{b}}$ as obtained in Experiment $\mathrm{I}$ in the present study. For a lead-lag delay of $16 \mathrm{~ms}$ Parham et al. found nearly identical responses to lead and lag for a full recovery at a delay of $40 \mathrm{~ms}$. ABR data as obtained from Experiment I in this study suggest a lead-lag delay of only $5.16 \mathrm{~ms}$ for a full recovery which is markedly smaller than predicted from these physiological results. 
Different lead-lag delays for a $50 \%$ recovery were found by Fitzpatrick et al. (1999) who determined recovery curves for several structures of the ascending auditory pathway in animals. These structures were the auditory nerve (AN) and the anteroventral cochlear nucleus (AVCN) of the cat and the superior olivary complex (SOC), the IC and the auditory cortex (AC) of the rabbit. On early stages of the auditory pathway (AN, AVCN, SOC) Fitzpatrick et al. found a 50\% recovery for a lead-lag delay around $2 \mathrm{~ms}$. In neurons of the IC a $50 \%$ recovery was found for lead-lag delays that average around $7 \mathrm{~ms}$, for neurons of the AC they are about $20 \mathrm{~ms}$. Overall, these values are smaller than those found by Parham et al. (1996).

Wickesberg and Oertel (1990) investigated monaural echo suppression mechanisms in mice $\mathrm{CN}$. They determined afferent fields with similar patterns of several AVCN neurons in the dorsal cochlear nucleus (DCN). Both bushy and stellate cells in the AVCN were inhibited by the activation of respective DCN neurons, i.e., all cells tested were targets of inhibitory input from the DCN. Moreover, Wickesberg and Oertel show that the locations of the afferent fields in the DCN follow the tonotopic organization of the cochlear nucleus complex.

Overall, their results suggest that action potentials in auditory nerve fibers evoke IPSPs that can suppress later inputs from auditory nerve fibers. They found a maximal suppression when the interval between signals in the auditory nerve fibers was $2 \mathrm{~ms}$; at smaller intervals the suppression has not developed and at larger intervals the suppression is less than maximal. In another study Wickesberg (1996) examined responses to pairs of clicks from single units in the VCN of anesthetized chinchillas. The response to the second click was suppressed for interclick intervals of 1 and $2 \mathrm{~ms}$, but was only little suppressed for an interclick interval of $4 \mathrm{~ms}$. However, responses of single neurons can only roughly be transformed into a prediction of evoked potentials that describe the average activation of neuron populations.

In the ABRs of the present study the amplitude of wave $V_{\mathrm{b}}$ for a lead-lag delay of $2 \mathrm{~ms}$ is reduced compared to that for a lead-lag delay of $5 \mathrm{~ms}$ which agrees with the monaural echo suppression mechanism found by Wickesberg and Oertel (1990). But previous psychophysical studies as well as the results of the present psychoacoustical experiments show that the precedence effect is strongest for lead-lag delays of about $5 \mathrm{~ms}$. For these lead-lag delays, however, only little monaural echo suppression was found (Wickesberg, 1996). If the precedence effect would appear in ABRs one might expect to see decreased amplitudes of wave $V_{\mathrm{b}}$ for a lead-lag delay of $5 \mathrm{~ms}$ compared to the amplitudes of wave $V_{\mathrm{b}}$ for a lead-lag delay of $20 \mathrm{~ms}$. As described in Section 3.1 this is not the case. All amplitudes of wave
$V_{\mathrm{b}}$ are fairly identical, i.e., no suppression effect was found in ABRs for a lead-lag delay of $5 \mathrm{~ms}$. Rather, a monotonous recovery of wave $V_{\mathrm{b}}$ is observed as the lead-lag delay increases which is consistent with physiological studies at the auditory nerve and at brainstem level (Fitzpatrick et al., 1999). ABRs reflect stimulus features (limited by recovery mechanisms) rather than the perception of the stimulus. As Fitzpatrick et al. found an increasing suppression characteristic on later stages of the ascending auditory pathway one might find suppression effects with longer durations on later stages. This is investigated by recording CAEPs using the MMN paradigm (see Section 3.4).

As shown in Fig. 4 latency differences between both waves $V$ are slightly larger than the lead-lag delay of the stimulus. This discrepancy of about $0.4 \mathrm{~ms}$ suggests that for the lead-lag delays employed in Experiment I both the first and the second wave $V$ were not elicited completely independently, although their amplitudes were very similar. However, this discrepancy is expected to disappear for larger lead-lag delays than those employed in this study because for very large lead-lag delays responses to lead and lag are assumed to be evoked independently. This assumption is confirmed by the fact that in Experiment II, in the diotic stimulation condition, where a lead-lag delay of $20 \mathrm{~ms}$ was employed (see Fig. 5), the latency difference between both waves $V$ is only $0.11 \mathrm{~ms}$ larger than the lead-lag delay of the stimulus.

Although the results of the current paper suggest that the early stages of the auditory pathway are not involved in the precedence effect, there are other studies that seem to show contradicting results. For example, Litovsky et al. (2002) described a patient suffering from a lesion including the IC. Psychoacoustical experiments of this particular patient showed a decreased precedence effect compared to that of control subjects. This finding suggests that the integrity of the IC is necessary for the occurrence of the precedence effect. As the brainstem potential component wave $V$ is assumed to be generated by the output of the superior olive, i.e., the vertical current over the lemniscus lateralis into the IC, one might expect to observe influences of the precedence effect in the amplitude or latency of this component. This is not the case according to the results of this study. Moreover, other studies demonstrate the involvement of cortical areas in the generation of the precedence effect (Hochster and Kelly, 1981; Cornelisse and Kelly, 1987). Liebenthal and Pratt (1999) mentioned several psychophysical experiments showing the influence of ongoing auditory information on echo-detectibility and sound localization. The dynamic aspect and complexity of the precedence effect have led investigators to suggest the involvement of high cognitive processing in the effect (Clifton, 1987; Saberi and Perrott, 1990; Freyman et al., 1991; Blauert, 1997). 


\subsubsection{Experiment II}

In Experiment II of the ABR recordings dichotic stimuli were applied in order to determine the influence of a lag-ITD of $300 \mu$ s on the ABRs. The differences between the first and second wave $V$ using dichotic stimuli (lag-ITD $=300 \mu \mathrm{s}$ ) were significantly increased by 0.11 and $0.15 \mathrm{~ms}$ (for lead-lag delays of 5 and $20 \mathrm{~ms}$, respectively) compared with the corresponding differences using diotic stimuli (lag-ITD $=0 \mu \mathrm{s}$ ). About half of the interaural delay of the stimulus is reflected in the ABRs. This finding is in accordance with the double delay-line coincidence detection model proposed by Jeffress (1948).

There are no significant differences of the amplitudes of wave $V_{\mathrm{b}}$ between lead-lag delays of 5 and $20 \mathrm{~ms}$. Indeed, amplitudes of all waves $V$, elicited by lead or lag, are nearly identical for all lead-lag delays. No suppression effect of wave $V_{\mathrm{b}}$ for a lead-lag delay of $5 \mathrm{~ms}$ is observable. Amplitudes of wave $V_{\mathrm{b}}$ obtained by dichotic stimulation are identical to those obtained by diotic stimulation. A suppression effect could be presumed as results of the psychoacoustical measurements showed increased discrimination thresholds for a lead-lag delay of $5 \mathrm{~ms}$. Hence, psychoacoustical data do not correlate with the amplitude characteristics of the second wave $V$.

Litovsky et al. (2001) and Litovsky and Delgutte (2002) showed that responses of single neurons of the IC in cats to lead and lag strongly depend on the lead azimuth for short lead-lag delays. Hence, one could argue that the amplitude of the second wave $V$ as obtained in this study may also depend on the azimuth, i.e., the lag-ITD. However, ABRs from the present study do not contradict these physiological data. Although, most responses of single neurons are dependent on the directions of lead and lag, the overall number of neurons that respond to lead and lag may be rather independent of the speaker positions. Since ABRs as obtained in the present study reflect the summing potential, i.e., the compound activity of all neurons in the far-field, no amplitude effect may occur.

\subsection{Comparison of $M M N$ data with related studies}

CAEP recordings using stimuli that evoke the precedence effect have not been reported before. However, results of the present study can be related to previous findings.

Systematic differences between 'good performers' (both in MMN tasks and related psychoacoustical tasks) and 'poor performers' were previously reported by Lang et al. (1990). In their psychoacoustical tasks pitch discrimination thresholds were determined and compared to MMN amplitudes determined with the same stimuli. The standard pitch was $698 \mathrm{~Hz}$ and deviant pitches varied between 710 and $2094 \mathrm{~Hz}$. Subjects with 'poor' pitch discrimination performances showed significantly smaller MMN components than those subjects that showed a 'moderate' or 'good' discrimination performance. MMN latencies decrease with increasing differences between standard and deviant pitch whereas MMN amplitudes increase.

In the present study, just noticeable changes of any sound feature were evaluated. MMN amplitudes are in agreement with the amplitude characteristics found by Lang et al., i.e., subjects that show a poor performance in the psychoacoustical tasks also show small MMN components. Hence, in both studies the MMN amplitudes are good predictors for discrimination performances. In contrast, latency characteristics of both studies do not agree: as shown in Fig. 7B not only the mean MMN amplitudes but also the mean latencies of the MMN for different lead-lag delays differ significantly. Latencies obtained with lead-lag delays of 1 and $20 \mathrm{~ms}$ are significantly larger than latencies obtained with a lead-lag delay of $5 \mathrm{~ms}$. Lang et al. found decreasing latencies for increasing MMN components. It is not clear if this discrepancy between both studies is due to the different stimuli and tasks employed or due to other factors. A systematic variation of the lag-ITD and leadlag delay would show in more detail how amplitude and latency of the MMN component are connected to these two parameters.

Schröger (1996) performed MMN recordings with lateralized stimuli. He applied a $900 \mathrm{~Hz}$ sinusoidal tone and a tone complex consisting of a 600 and a $3000 \mathrm{~Hz}$ component. Standard stimuli had no ITD whereas deviant stimuli had an ITD of $300 \mu$ s. For electrode Fz (referenced to the nose) MMN peak latencies for both stimuli were found between 135 and $170 \mathrm{~ms}$ whereas MMN peak-to-peak amplitudes were about 2.2 and $4 \mu \mathrm{V}$ for the sinusoidal tone and the tone complex, respectively.

MMN components in the present study were recorded using double click-pairs and a lag-ITD of $800 \mu \mathrm{s}$. For lead-lag delays of 1,5 and $20 \mathrm{~ms}$ MMN components of the same electrode peak at latencies of 165,138 and $154 \mathrm{~ms}$ with peak-to-peak amplitudes of $1.58,0.88$ and $1.12 \mu \mathrm{V}$, respectively. Thus, similar MMN latencies appear in both studies, although the ITD used by Schröger is much smaller $(300 \mu s)$ than that of the present study $(800 \mu \mathrm{V})$. MMN amplitudes of the present study are smaller than those found by Schröger. Psychoacoustic measurements of the present study show that discrimination thresholds are elevated for non-zero lead-lag delays compared to the zero lead-lag delay condition. Hence, one reason for the differences between the MMN amplitudes of both studies may be the suppressive effect of the lead click used in the present study.

The physiological correlate of the precedence effect seen in our MMN recordings agrees qualitatively with the recovery curves predicted by Fitzpatrick et al. (1999). In their physiological study, neurons of different 
stages of the auditory pathway of the cat were examined, e.g., single neurons of the IC and the auditory cortex. Due to slow recoveries exhibited by neurons of the IC and especially the auditory cortex, they predict a dominance of information provided by the lead compared to the information provided by the lag for lead-lag delays up to $300 \mathrm{~ms}$. Latencies of the MMN components as found in the results of the current study are clearly within this interval. This confirms the fact that the suppressive influence of the lead is still observable at these late stages of the auditory pathway like the auditory cortex.

\subsection{Relation between discrimination performance and MMN amplitudes}

Cortical auditory evoked potentials were recorded according to the paradigm of the MMN presenting frequent reference (lag-ITD $=0 \mu \mathrm{s})$ and rare deviant stimuli (lag-ITD $=800 \mu \mathrm{s}$ ) for lead-lag delays of 1, 5 and $20 \mathrm{~ms}$, respectively. Subjects could be separated into two groups according to their recorded potentials. Hardly any MMN component appears in subjects of group A whereas consistent MMN components are observable in frontal channels in subjects of group B. Results of the CAEP recordings of both groups can be related to the respective performances in the psychoacoustical tasks. Results of the CAEP recordings show that the MMN was elicited by the lag-ITD of $800 \mu \mathrm{s}$ in those subjects that are able to discriminate the deviant from reference stimuli in the psychophysical tasks. That is, for subjects of group B, the MMN recordings were made at above threshold level for all three lead-lag delays. In contrast, for subjects of group A, MMN recordings for a lead-lag delay of $5 \mathrm{~ms}$ were performed at a level below threshold while for lead-lag delays of 1 and $20 \mathrm{~ms}$ they were made near threshold level. An absolute discrimination threshold may be determined by varying the lag-ITD of the deviant as the occurrence of an MMN component is correlated with a discriminable change of the stimulus. Therefore, below the discrimination threshold no $\mathrm{MMN}$ is expected to be elicited whereas above the discrimination threshold an MMN component should be observable.

Generally, in subjects of group B, data sets of psychoacoustical and CAEP measurements show a high correlation, i.e., the relation between the discrimination thresholds obtained in the psychophysical experiments equals the inverse relation of the MMN amplitudes of the CAEP recordings. It seems that the amplitude of the MMN component reflects the detectability of the change between reference and target stimulus rather than directly any stimulus feature. Psychophysical discrimination performances were influenced by the precedence effect which is activated if two sounds are presented in close succession. As the amplitudes of the
MMN components show a high correlation with the discrimination performances of the behavioral tasks it can be stated that a neurophysiological correlate for the precedence effect was found on the level of the CAEP.

\section{Summary and conclusions}

The characteristics of the ABRs as recorded in this study are similar to the characteristics of neural response patterns on early stages of the auditory path way. In both cases the first click of a double click-pair evokes a recovery state of the neurons. Fifty percent recovery values found in physiological studies are comparable to the value of $3.28 \mathrm{~ms}$ obtained in the present study.

ABRs of this study rather reflect features of the stimulus than its perception. Applying double click-pairs with a lead-lag delay of $5 \mathrm{~ms}$, two waves $V$ of equal amplitude were observed on the early stages of the auditory pathway. Amplitudes and latencies of the ABRs were not influenced by the precedence effect as a change of the directional information (lag-ITD) was not suppressed, i.e., the second wave $V$ was not reduced in amplitude applying a lead-lag delay of $5 \mathrm{~ms}$ and a lagITD of $300 \mu \mathrm{s}$. Additionally, the embedded ITD in the stimulus can also be observed in the potentials. This means, that directional information provided by a lagITD is not suppressed on the early stages of the auditory pathway but is reflected in the ABRs and therefore available to the auditory system on this level.

On the later stages of the auditory pathway, the characteristics of the recorded potentials, i.e., the MMN, reflect the perception of the stimulus rather than its features as these potentials relate to the performances obtained from psychoacoustical tasks. These findings suggest that the precedence effect is not the result of an insufficient accuracy of the peripheral processing.

In the subgroup of five listeners that performed sufficiently well in the corresponding psychoacoustical task, a close correlation was found between the amplitude of the MMN components and the respective discrimination threshold obtained from the psychoacoustical procedure. This agreement can be interpreted as a neural correlate of the precedence effect in humans.

\section{References}

Blauert, J., 1997. Spatial Hearing: The Psychophysics of Human Sound Localization. The MIT Press, Cambridge, MA.

Burkard, R., 1991. Human brain-stem auditory evoked responses obtained by cross correlation to trains of clicks, noise bursts, and tone bursts. J. Acoust. Soc. Am. 90 (3), 1398-1404.

Burkard, R., Shi, Y., Hecox, K.E., 1990. A comparison of maximum length and Legendre sequences for the derivation of brain-stem auditory-evoked responses at rapid rates of stimulation. J. Acoust. Soc. Am. 87 (4), 1656-1664. 
Clifton, R.K., 1987. Breakdown of echo suppression in the precedence effect. J. Acoust. Soc. Am. 82 (5), 1834-1835.

Cornelisse, L.E., Kelly, J.B., 1987. The effect of cerebrovascular accident on the ability to localize sounds under conditions of the precedence effect. Neurophysiologia 25 (2), 449-452.

Eysholdt, U., Schreiner, C., 1982. Maximum length sequences - a fast method for measuring brain-stem-evoked responses. Audiology 21 (3), 242-250.

Fitzpatrick, D.C., Kuwada, S., Batra, R., Trahiotis, C., 1995. Neural responses to simple simulated echoes in the auditory brain stem of the unanesthetized rabbit. J. Neurophysiol. 74 (6), 2469-2486.

Fitzpatrick, D.C., Kuwada, S., Kim, D.O., Parham, K., Batra, R., 1999. Responses of neurons to click-pairs as simulated echoes: auditory nerve to auditory cortex. J. Acoust. Soc. Am. 106 (6), 3460-3472.

Freyman, R.L., Clifton, R.K., Litovsky, R.Y., 1991. Dynamic processes in the precedence effect. J. Acoust. Soc. Am. 90 (2), 874-884.

Hartung, K., Trahiotis, C., 2001. Peripheral auditory processing and investigations of the 'precedence effect' which utilize successive transient stimuli. J. Acoust. Soc. Am. 110 (3), 1505-1513.

Hey, M., 2001. Kanaltrennung bei hochratiger sequentieller pulsatiler Elektrostimulation der Cochlea. Ph.D. thesis, C. v. O. University of Oldenburg.

Hochster, M.E., Kelly, J.B., 1981. The precedence effect and sound localization by children with temporal lobe epilepsy. Neurophysiologia 19, 49-55.

Jeffress, L.A., 1948. A place theory of sound localization. J. Comp. Physiol. Psychol. 41, 35-39.

Keller, C.H., Takahashi, T.T., 1996. Responses to simulated echoes by neurons in the barn owl's auditory space map. J. Comp. Physiol. 178 (4), 499-512.

Lang, A.H., Nyrke, T., Ek, M., Aaltonen, O., Raimo, I., Näätänen, R., 1990. Pitch discrimination performance and auditive eventrelated potentials. In: Brunia, C.H.M (Ed.), Psychophysiological Brain Research, vol. 1, pp. 294-298.

Liebenthal, E., Pratt, H., 1997. Evidence for primary auditory cortex involvement in the echo suppression precedence effect: a 3CLT study. J. Basic Clin. Physiol. Pharmacol. 8 (3), 181-201.

Liebenthal, E., Pratt, H., 1999. Human auditory cortex electrophysiological correlates of the precedence effect: binaural echo lateralization suppression. J. Acoust. Soc. Am. 106 (1), 291-303.

Litovsky, R.Y., 1998. Physiological studies of the precedence effect in the inferior colliculus of the kitten. J. Neurophysiol. 103 (6), 3139-3152.

Litovsky, R.Y., Colburn, H.S., Yost, W.A., Guzman, S.J., 1999. The precedence effect. J. Acoust. Soc. Am. 106 (4), 1633-1654.

Litovsky, R.Y., Delgutte, B., 2002. Neural correlates of the precedence effect in the inferior colliculus: effect of localization cues. J. Neurophysiol. 87 (2), 976-994.

Litovsky, R.Y., Fligor, B.J., Tramo, M.J., 2002. Functional role of the human inferior colliculus in binaural hearing. Hear. Res. 165, 177188.

Litovsky, R.Y., Lane, C.C., Atenicio, C., Delgutte, B., 2001. Physiological measures of the precedence effect and spatial release from masking in the cat inferior colliculus. In: Houtsma, A.J.M., Kohlrausch, A., Prijs, V.F., Schoonhoven, R. (Eds.), Physiological and Psychophysical Bases of Auditory Function. Shaker Publishing, Maastricht, The Netherlands.

Litovsky, R.Y., Rakerd, B., Yin, T.C., Hartmann, W.M., 1997. Psychophysical and physiological evidence for a precedence effect in the median sagittal plane. J. Neurophysiol. 77 (4), 2223-2226.

Litovsky, R.Y., Yin, T.C., 1998. Physiological studies of the precedence effect in the inferior colliculus of the cat. I. Correlates of psychophysics. J. Neurophysiol. 80 (3), 1285-1301.

Møller, A.R., Jannetta, P.J., 1982. Evoked potentilas from the inferior culliculus in man. Electroencephalogr. Clin. Neurophysiol. 53 (6), 612-620.

Møller, A.R., Jannetta, P.J., Jho, H.D., 1994. Click-evoked responses from the cochlear nucleus: a study in human. Electroencephalogr. Clin. Neurophysiol. 92 (3), 215-224.

Näätänen, R., Gaillard, A.W.K., Mäantysalo, S., 1978. Early selectiveattention effect on evoked potential reinterpreted. Acta Psychol. 42, 313-329.

Parham, K., Zhao, H.B., Kim, D.O., 1996. Responses of auditory nerve fibers of the unanesthetized decerebrate cat to click pairs as simulated echoes. J. Neurophysiol. 76 (1), 17-29.

Riedel, H., Granzow, M., Kollmeier, B., 2001. Single-sweep-based methods to improve the quality of auditory brain stem responses, Part II: Averaging methods. Zeitschrift für Audiologie/Audiol. Acoust. 40 (2), 62-85.

Riedel, H., Kollmeier, B., 2002. Auditory brain stem responses evoked by lateralized clicks: is lateralization extracted in the human brainstem? Hear. Res. 163, 12-26.

Saberi, K., Perrott, D.R., 1990. Lateralization thresholds obtained under conditions in which the precedence effect is assumed to operate. J. Acoust. Soc. Am. 87 (4), 1732-1737.

Scherg, M., von Cramon, D., 1985. A new interpretation of the generators of BAEP waves I-V: results of a spatio-temporal dipole model. Electroencephalogr. Clin. Neurophysiol. 62 (4), 290-299.

Schröger, E., 1996. Interaural time and level differences: integrated or separated process. Hear. Res. 96, 191-198.

Tollin, D.J., Yin, T.C., 2003. Psychophysical investigation of an auditory spatial illusion in cats: the precedence effect. J. Neurophysiol. 90 (4), 2149-2162.

Wallach, H., Newman, E.B., Rosenzweig, M.R., 1949. The precedence effect in sound localization. Am. J. Psychol. LXII (3), 315-336.

Wickesberg, R., 1996. Rapid inhibition in the cochlear nuclear complex of the chinchilla. J. Acoust. Soc. Am. 100 (3), 1691-1702.

Wickesberg, R.E., Oertel, D., 1990. Delayed, frequency-specific inhibition in the cochlear nuclei of mice: a mechanism for monaural echo suppression. J. Neurosci. 10 (6), 1762-1768.

Yin, T.C., 1994. Physiological correlates of the precedence effect and summing localization in the inferior colliculus of the cat. J. Neurosci. 14 (9), 5170-5186.

Zurek, P.M., 1980. The precedence effect and its possible role in the avoidance of interaural ambiguities. J. Acoust. Soc. Am. 67 (3), 953-964. 